\title{
Die SGTV -
}

\section{wieder bei den Hausärzten}

\section{Dominik Heima ${ }^{\text {, Myriam Oberleb }}{ }^{\text {, Thomas S. Müller }}{ }^{a, c}$}

a PD Dr. med., Vorstand Schweizerische Gesellschaft für Traumatologie und Versicherungsmedizin; ' ${ }^{b}$ Dr. med., Praxis Jenaz, Fortbildungsverantwortliche grisomed, Fortbildungsdelegierte SGAIM; ' Dr. med., Chefarzt Interdisziplinäre Notfallstation, Kantonsspital Graubünden

Am 16. Januar 2019 fand seit vielen Jahrzehnten wieder einmal ein Workshop der Schweizerischen Gesellschaft für Traumatologie und Versicherungsmedizin SGTV statt. In Zusammenarbeit mit dem Bündner Ärzteverein und grisomed wurde in Chur eine unfallchirurgische Weiterbildung für Hausärzte veranstaltet. Das Interesse war gross, und die Rückmeldungen waren ausnahmslos positiv (eine Teilnahmebescheinigung mit den Credits gab es nur gegen einen ausgefüllten Fragebogen zum Anlass!).

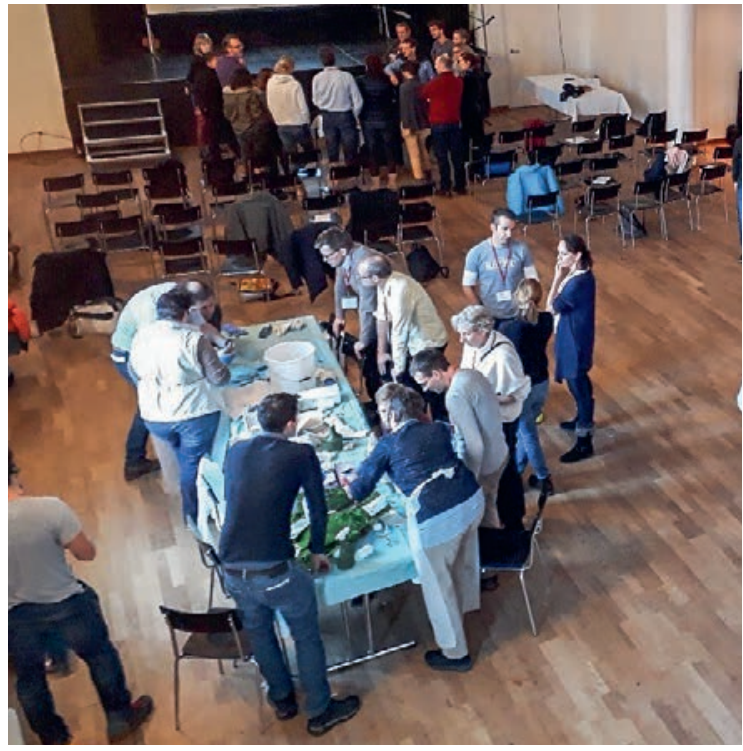

Abbildung 1: Praktische Übungen im Saal am Nachmittag.

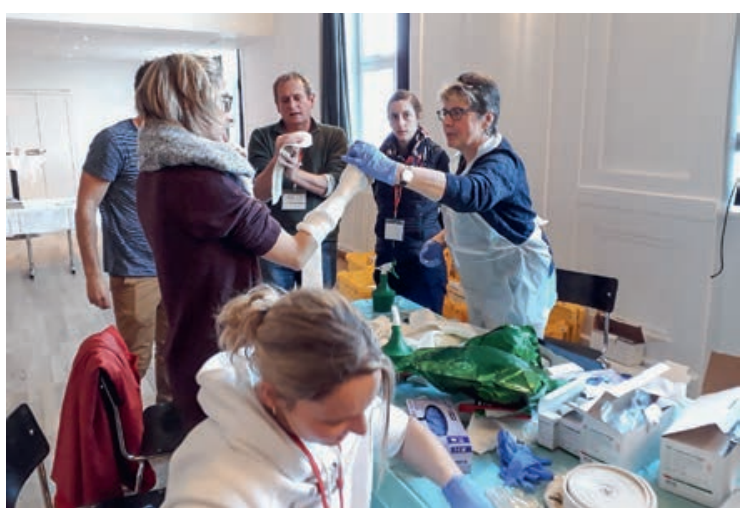

Abbildung 2: Ist es wirklich die Intrinsic-plus-Stellung?
Mehr als hundert Jahre nach ihrer Gründung am 7. Januar 1912, damals unter dem Namen «Schweizer Unfallärzte», veranstaltete die SGTV zusammen mit weiteren Partnern wieder einen traumatologischen Workshop, zu dem die Hausärzte des Kantons Graubünden und der angrenzenden Regionen eingeladen waren. Damals, bei der Gründung der Gesellschaft zu Beginn des 20. Jahrhunderts, wurde bemängelt, dass «die Ausbildung des praktischen Arztes in der Behandlung der Unfallverletzungen sehr mangelhaft sei» [1]. Heute, viele Jahrzehnte später, ist eine ähnliche Entwicklung wieder zu spüren. In der Weiterbildung zum Facharzt Allgemeine Innere Medizin ist das Fach Chirurgie nicht mehr gefordert, man kann, muss aber darin nicht «famuliert» haben. Die traumatologische Kompetenz unter den (jungen) Hausärzten nimmt ab [2]. In den städtischen Regionen wird vermehrt auf die Notfallstationen der Spitäler verwiesen/zugewiesen, was dann dort zur Ressourcen-Verknappung führt. In der Peripherie (unter anderem auch in Touristenorten) mit längerem Anfahrtsweg bis zum nächsten Spital wird dies zu einem zunehmenden Problem für die dortige Bevölkerung und die Gäste.

Im Rahmen eines Pilotprojektes wurde deshalb in Chur, im Saal Marsöl, ein erster traumatologischer Workshop zum Thema «Hand- und Handgelenksverletzungen» durchgeführt. Die Struktur der Veranstaltung beinhaltete einen Morgen mit Vorträgen, eine kurze Mittagspause und am Nachmittag praktische Übungen im Rotationssystem, also Theorie und angewandte Praxis. In den Vorträgen war der Fokus auf die Diagnostik, die konservativen Behandlungsmethoden 


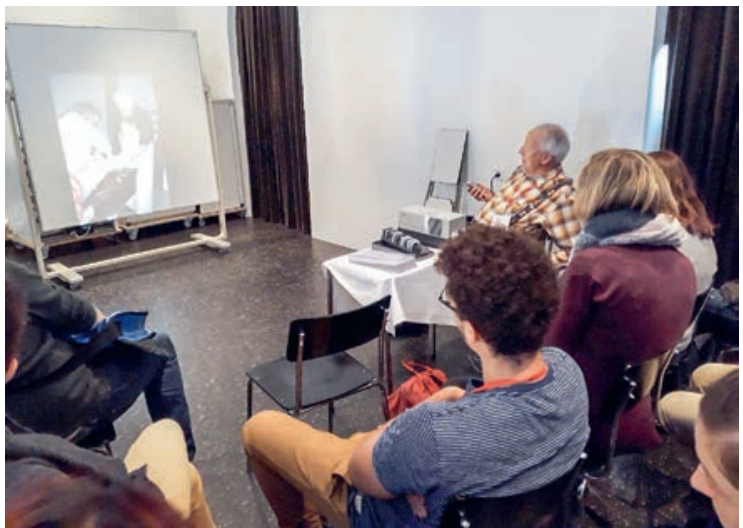

Abbildung 3: Die Fallsammlung von und mit Dr. Röthlisberger.

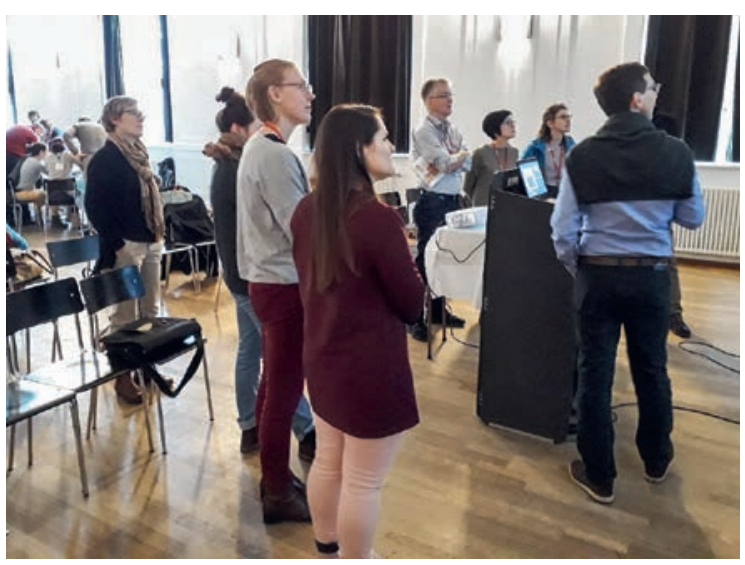

Abbildung 4: Falldiskussion.

und die Entscheidung zur konservativen oder operativen Therapie gelegt: «Ich habe viel gelernt und Sicherheit bekommen in der Entscheidung: konservativ-operativ", notierte eine Teilnehmerin auf dem Fragebogen. Auch auf das Thema einer genauen Dokumentation bei der ersten Arztkonsultation sowie einer korrekten Beurteilung der Arbeitsunfähigkeit wurde grossen Wert gelegt. Denn wie die Versicherungsmedizinerin darlegte, erlauben nur genaue Angaben zum Unfallmechanismus und zu den vorliegenden Verletzungen eine versicherungsmedizinische Beurteilung hinsichtlich Unfall oder Krankheit (Zuständigkeit der entsprechenden Kostenträger), bei Gerichtsverfahren und auch zum Beispiel bei der Frage nach einem Behandlungsfehler.

Am Nachmittag wurde gearbeitet: In Rotation an vier Stationen wurde unter kundiger Anleitung und mit viel Begeisterung - auf Instruktorinnen- und Teilnehmer(innen)seite - gegipst und wurden volare Handgelenksschienen, Daumenkänel und weitere einfache Immobilisationen geübt. Bei der ergotherapeutischen Station wurden Tipps und Tricks zur Herstellung von kleinen Schienen, von Tapeverbänden und weiteren Hilfsmitteln weitergegeben und mit dem Material eines Sponsors und kundiger Anleitung durch lokale Ergotherapeuten/-innen auch selbst ausprobiert. Falldemonstrationen anhand von Röntgenbildern animierten zur Diskussion von Diagnose und Behandlung der Verletzung. Am meisten Applaus bekam der «Postenchef» des vierten Postens, Dr. med. Dr. h.c. Tino Röthlisberger, über 80-jährig, aus Arosa: Er zeigte mit Diapositiven (!) unter dem Titel «Die Fallsammlung des 'Alten': pure Erfahrung» eine Auswahl seiner vielen klinischen Bilder; es war eine Reise zurück in die Vergangenheit, wo mit den damaligen Mitteln die Unfallverletzungen kompetent, einfach und billig behandelt werden konnten. Die Teilnehmer/-innen lobten diese praktischen Übungen als "wichtig», "gute Beziehung zur Theorie am Morgen", "sehr hilfreich" in ihren Kommentaren. Und noch etwas fiel auf in den Bewertungen zur Frage, ob der Kurs wichtig sei: «Ja, auch zum Kennenlernen unter Ärzten sehr wertvoll», schrieb eine Teilnehmerin. Diesem Aspekt gerecht wurde auch das kurze, aber äusserst gesprächige (Lärmpegel) Mittagessen im gemütlichen «Marsölstübli».

38 Hausärzte (und angehende) haben diesen Pilotkurs besucht - ihre Rückmeldungen reichen von «sehr praxisnah" über "sehr wichtig» bis zu "positiv überrascht» (!). Weitere Kurse zu anderen Themen (Sprunggelenk, Schulter, Ellbogen, Wundversorgung ...) im gleichen Rahmen werden befürwortet. Der Mix von Theorie und Praxis wird als ausgewogen beurteilt. Einer Erweiterung auf zwei Tage steht man aber eher skeptisch gegenüber. Angesichts dieser Resonanz spricht man von einem weiteren Kurs im Herbst in Luzern, und auch die Ärzteschaft im Aargau hat ihr Interesse angemeldet. Ganz wichtig sind sicher der Einbezug der Ärzte der lokalen Spitäler, die die Teilnehmer bei einem solchen Kurs kennenlernen können, und der paramedizinischen Bereiche wie Physiotherapie/Ergotherapie sowie die Mitarbeit der kantonalen Ärzteorganisationen und ansässigen Hausärzte. Und die SGTV ist wieder da, wo sie einmal war, in ihrer Aufgabe in der Ausbildung zur Unfallmedizin.

«Danke vielmals, hat Spass gemacht, gute Gruppendynamik», schrieb eine 40-jährige Teilnehmerin. Einzig der «Saal war leider etwas kalt», meinte jemand anderes ...

Bildnachweise

Fotos Dominik Heim

\section{Literatur}

1 Baur E. Aus der Geschichte der Schweizerischen Gesellschaft für Unfallmedizin und Berufskrankheiten (es existieren 3 Exemplare) 1993: S. 4.

2 Aschwanden E. Weshalb Hausärzte Patienten mit einem Bruch ins Spital schicken. NZZ.1.12.2017. 